\title{
A Framework for Using Diffusion Weighted Imaging to Improve Cortical Parcellation ${ }^{\star}$
}

\author{
Matthew J. Clarkson ${ }^{1,2}$, Ian B. Malone ${ }^{2}$, Marc Modat ${ }^{1}$, Kelvin K. Leung ${ }^{2,1}$, \\ Natalie Ryan ${ }^{2}$, Daniel C. Alexander ${ }^{1}$, Nick C. Fox ${ }^{2}$, and Sébastien Ourselin ${ }^{1,2}$ \\ 1 Centre for Medical Image Computing, University College London, WC1E 6BT, UK \\ 2 Dementia Research Centre, UCL Institute of Neurology, London, WC1N 3BG, UK
}

\begin{abstract}
Cortical parcellation refers to anatomical labelling of every point in the cortex. An accurate parcellation is useful in many analysis techniques including the study of regional changes in cortical thickness or volume in ageing and neurodegeneration. Parcellation is also key to anatomic apportioning of functional imaging changes. We present preliminary work on a novel algorithm that takes an entire cortical parcellation and iteratively updates it to better match connectivity information derived from diffusion weighted imaging. We demonstrate the algorithm on a cohort of 17 healthy controls. Initial results show the algorithm recovering artificially induced mis-registrations of the parcellation and also converging to a group-wise average. This work introduces a framework to investigate the relationship between structure and function, with no a-priori knowledge of specific regions of interest.
\end{abstract}

\section{Introduction}

Cortical parcellation is a labelling of every point in the cortex [5]. This labelling delineates regions throughout the cortex, enabling subsequent analysis on a perregion basis, and is an essential tool for neuroimaging research. An accurate cortical parcellation is of use when analysing properties such as thickness [4] or white matter connectivity [7. As the parcellation is a key behind-the-scenes component, it is essential for understanding the underlying anatomy and both the cross-sectional and longitudinal changes that occur in neurodegenerative diseases.

Using magnetic resonance imaging (MRI), previous parcellation methods have looked at labelling parts of the cortex, such as prominent sulci, to sub-divide the volume of each hemisphere [10], or using watershed techniques to label all sulci [8]. Subsequently, Fishl et. al. [5] developed a comprehensive method to label

\footnotetext{
* This work was undertaken at UCL/UCLH which received a proportion of funding from the Department of Health's NIHR Biomedical Research Centres funding scheme. The Dementia Research Centre is an Alzheimer's Research Trust Coordinating centre. KKL is supported by TSB grant M1638A. MC is supported by TSB grant M1638A and UCLH/UCL CBRC grant 168. NCF is funded by the Medical Research Council (UK).
} 
all voxels in the cortex. This method, based on a probabilistic atlas derived from manually labelled datasets is incorporated into the widely used FreeSurfer software package.

However, it is known that the topographical boundaries in a cortical parcellation may not be visible in structural MRI scans and only have a limited correspondence to underlying cytoarchitecture [1. Recently it has been shown that connectivity profiles derived from diffusion data can elucidate functionally distinct regions in the cortex [6]2. These methods infer a parcellation directly from diffusion weighted imaging (DWI) data by clustering together voxels with similar connectivity. Thus far, these voxel based methods have been applied to regions of the brain such as the supplementary motor cortex (SMA) and preSMA [6] and also to define 9 subregions of the cingulate cortex [2. Most recently, surface based methods have been developed to register brains based on regional connectivity [9] and also analyse which areas have sufficient connectivity to drive a parcellation [3. We describe work that combines DWI and volumetric MRI data to modify an existing parcellation of the whole cortex, with the hypothesis that a parcellation based on functional connectivity provides a parcellation that is more functionally and cytoarchitecturally correct when compared with conventional topology driven parcellation. Importantly we first asked whether these approaches give concordant or different results, without pre-judging which is better since this judgement may be dependent upon the use to which the parcellation is put.

\section{Methods}

We first describe several pre-processing steps, before describing the proposed algorithm. For these experiments, we use the cortical parcellation, and spherical surface model produced by FreeSurfer 1 [5] and probabilistic tractography implemented in MRtrix [11, however, the proposed algorithm could be applied to any triangulated surface model representing the cortex with pointwise connectivity established. Furthermore, we pre-process our T1-weighted (T1w) images using the FreeSurfer pre-processing pipeline. The FreeSurfer pre-processing pipeline includes steps that linearly resample to $1 \mathrm{~mm}$ iso-tropic voxels, performs N3 intensity inhomogeneity correction, registers to Talairach space and then performs another white matter specific intensity normalisation before continuing on to perform a cortical thickness and volume based processing stream. These FreeSurfer pre-processing steps are not discussed as part of this paper.

\subsection{Combining Structural and Connectivity Information}

For each subject's T1w scan, we run the FreeSurfer cross sectional pipeline, and extract the triangulated white matter surface, the inflated spherical surface and the 36 anatomical labels, for both the left and right side of the brain.

\footnotetext{
${ }^{1}$ http://surfer.nmr.mgh.harvard.edu/fswiki

${ }^{2}$ http://www.brain.org.au/software/
} 


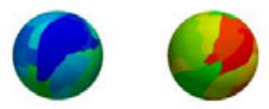

(a)

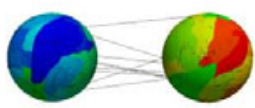

(b)

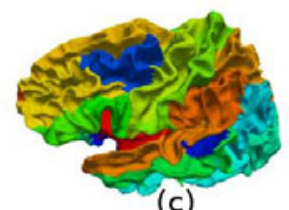

(c)

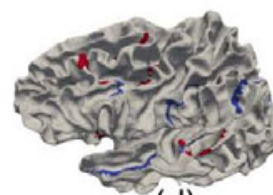

(d)

Fig. 1. Overview of method. (a) Spherical representations of the left and right cortical surfaces are extracted using FreeSurfer. (b) Connectivity information from MRtrix is added (only the first 500 tracts are shown). (c) The parcellation boundaries are optimised using the bi-spherical model, and then mapped back to the white matter surface. (d) The difference can be visualized, and the updated parcellation used in further cortical analysis.

FreeSurfer guarantees the ordering of points listed in the white matter surface matches the ordering of points in the corresponding inflated spherical surface, for each side, thereby enabling simple mapping between the two. The left and right spherical mesh models were combined into a single fil@ 3 , with each sphere offset by $\pm(100,100,100) \mathrm{mm}$ to aid visualization, and the left hemisphere labelled with anatomical regions 0-35, and the right hemisphere labelled with the same anatomical regions, but with labels 36-71, illustrated in Fig. 11(a).

For each subject's DWI scans, Fibre Orientation Distributions (FODs) were computed by Constrained Spherical Deconvolution with a maximum harmonic degree of eight[11], using the MRtrix package. Probabilistic tractography was performed by seeding 100,000 tracts within a cortical mask using MRtrix with default parameters. The tracts were then filtered to only store the endpoints of tracts that started and finished within the cortical mask (i.e. those that connect grey matter). Typically this resulted in circa. 70,000 tracts per subject.

The diffusion data was registered (9 degrees-of-freedom) to the T1w scans, and the tracts and FreeSurfer white matter surfaces transformed into T1w image space. For each end of each tract, we find the closest point in either the left or right white matter surface, and then the corresponding point in the left or right spherical models. Each tract is thus attached to the spherical model, and is illustrated in Fig. 1(b). In practice, the number of triangles in the white matter mesh is far greater than the number of tracts, leading to a sparsely populated model. Thus we subsample each spherical model, and re-attach the tracts to the nearest spherical surface point. The output of the pre-processing is a single bispherical model containing two labelled spheres representing the left and right cortical hemispheres and a large number of lines representing the connectivity, as determined from the tractography.

\subsection{Cortical Parcellation Optimisation Algorithm}

The algorithm we propose is a group-wise optimisation, designed to update each subject's cortical parcellation, to achieve consistency between subjects in the

\footnotetext{
${ }^{3}$ http://www.vtk.org
} 
within subject connectivity. We desire a mean connectivity profile to remove bias caused by any one individual. Implicitly, we are assuming that the group of subjects should have similar connectivity. This may not be the case under various disease conditions, so to investigate the framework, we concentrate on healthy controls.

Consider a dataset of $P$ points in $\mathbb{R}^{3}$, where with each point $\mathbf{p}_{i=1 \ldots P}$ we associate a single label $l_{j=1 \ldots L}$. Consider also that the dataset contains $T$ lines, denoted by $t_{k=1 \ldots T}$ where $t_{k}^{i, i^{\prime}}$ denotes the fact that the $k$-th line connects point $i$ with $i^{\prime}$ and $l\left(t_{k}^{i}\right)$ and $l\left(t_{k}^{i^{\prime}}\right)$ denote the label values associated with line $k$ at points $i$ and $i^{\prime}$ respectively. For a given configuration of labels, we can compute a symmetric $2 \mathrm{D}$ histogram $H$, where each bin $H\left(l_{j}, l_{j^{\prime}}\right)$ contains the number of label $l_{j}$ connected to label $l_{j^{\prime}}$ via the set of $T$ lines. Thus:

$$
H\left(l_{j}, l_{j^{\prime}}\right)=\frac{1}{T} \sum_{k=1}^{T} \begin{cases}1 & \text { if } l\left(t_{k}^{i}\right)=l_{j} \text { and } l\left(t_{k}^{i^{\prime}}\right)=l_{j^{\prime}} \\ 0 & \text { otherwise }\end{cases}
$$

Thus, for a given bi-spherical model (see Fig. 1(b)), we can compute a 2D histogram of label connectivity. Furthermore for $N$ 2D histograms of label connectivity, we define the mean connectivity histogram as

$$
\hat{H}\left(l_{j}, l_{j^{\prime}}\right)=\frac{1}{N} \sum_{n=1}^{N} H_{n}\left(l_{j}, l_{j^{\prime}}\right)
$$

Finally for two $2 \mathrm{D}$ histograms $H_{n}$ and $H_{n^{\prime}}$, we define a sum of squared difference histogram similarity measure as

$$
S\left(H_{n}, H_{n^{\prime}}\right)=\sum_{j=1}^{L} \sum_{j^{\prime}=1}^{L}\left(H_{n}\left(l_{j}, l_{j^{\prime}}\right)-H_{n^{\prime}}\left(l_{j}, l_{j^{\prime}}\right)\right)^{2}
$$

Algorithm 10utlines a single subject optimisation that matches a single subject's bi-spherical model to a label connectivity histogram. This could be used in a stand alone sense if the label connectivity histogram was known a-priori from another process.

The overall optimisation proceeds in a group-wise fashion, and is shown in Algorithm 2. For a group of $N$ bi-spherical models, an average connectivity histogram is calculated, and then for each subject, the parcellation is optimised using Algorithm 1 to drive towards the average. Within this combined framework, there are many alternatives that could be suggested, some of which are outlined in section 3 Fig. 1(c) and (d) show the labels mapped to the cortical surface, and after an update shows the difference image, illustrating updated region boundaries.

\subsection{Experiments and Results}

17 healthy control subjects were selected, and each scanned on a 3T Siemens TrioTim scanner, with a 32 channel head coil, using an MPRAGE sequence 


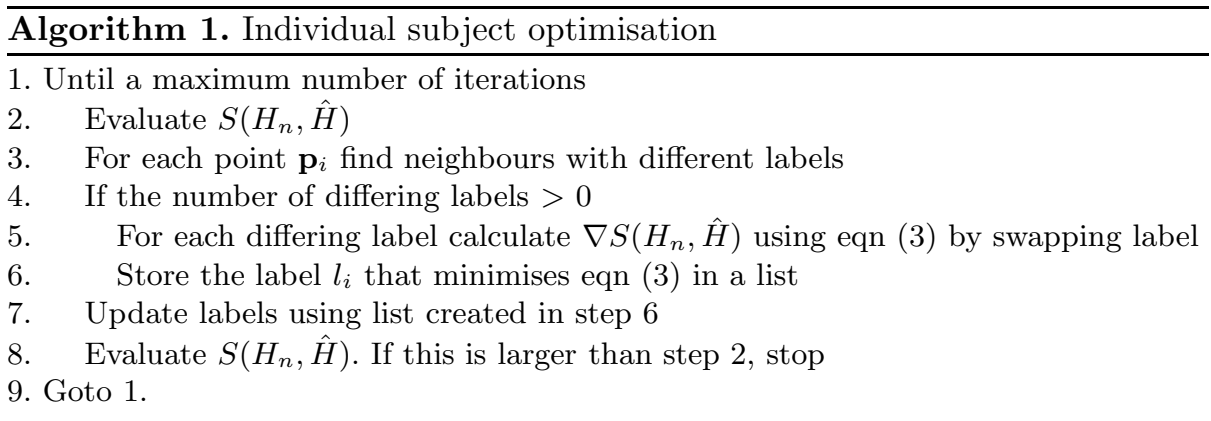

\section{Algorithm 2. Group-wise optimisation}

1. Until a maximum number of iterations

2. For each subject $n=1 \ldots N$ compute histogram $H_{n}\left(l_{j}, l_{j^{\prime}}\right)$ using eqn (1)

3. Compute the mean connectivity histogram using eqn (2)

4. For each subject $n=1 \ldots N$ optimise the parcellation values using Algorithm 1

5. Goto 1.

( $\mathrm{TE}=2.9 \mathrm{~ms}$, inversion interval $\mathrm{TR}=2200 \mathrm{~ms}, \mathrm{TI}=900 \mathrm{~ms})$. T1-weighted volumetric images were obtained with a $28.2-\mathrm{cm}$ field of view and $256 \times 256$ acquisition matrix to provide 208 continuous $1.1 \mathrm{~mm}$ thick slices. DWI images were obtained $\left(\mathrm{TE}=91 \mathrm{~ms}, \mathrm{TR}=6800 \mathrm{~ms}, 64\right.$ gradient directions, $\mathrm{b}=1000 \mathrm{smm}^{-2}$, and $8 \times B_{0}$ images) with a $24-\mathrm{cm}$ field of view and $96 \times 96$ acquisition matrix, providing 55 continuous $2.5 \mathrm{~mm}$ slices. FreeSurfer's cortical processing stream was run on each subject's T1 image, and MRtrix's tractography performed using a single $B_{0}$ image and 64 gradient directions.

For each of the 17 subjects, the histogram of label connectivity was generated. The labels were then mis-registered by selecting every other region (i.e. regions $0,2,4 \ldots 70)$ in turn, and updating the neighbours of each boundary point to that region label, similar to dilating a boundary in an intensity image. We did this $1,2 \ldots 5$ times, resulting in 5 datasets of 17 mis-labelled models. We then used Algorithm 1 to optimise the labels, on a per-subject basis, to match them to their original label connectivity histogram. We visually inspected the results. For all the levels of mis-registration, and for all subjects, the algorithm moved the boundaries of the regions. It could be seen that in some cases the apparent mis-registration increased. Even in this case, there is no gold standard, as the diffusion data may suggest a different parcellation to the initial topographical parcellation.

Fig. 2 shows a visualisation of the results for a single subject. Fig. 2(a) shows the bi-spherical model showing left and right hemispheres, with parcellation labels attached. Fig. 2(b) shows a difference image, the thick blue and red lines 


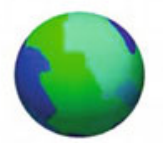

(a)
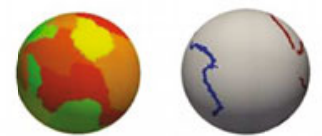

(b)

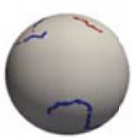

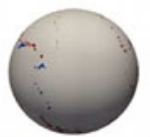

(c)

Fig. 2. Results of Algorithm 1 (a) Spherical representations of the left and right cortical surfaces. (b) A difference image showing the change in region label before running Algorithm 1 (c) A difference image showing the change in region label after running Algorithm 1 .

shows boundaries that have been moved. Fig. 2(c) shows the boundaries after the optimisation algorithm has run. It can be seen that Algorithm 1 has reduced the size of the thick blue and red lines, indicating that the algorithm converged back towards the original label connectivity histogram. Fig. 2(c) also shows that some noise has been introduced, most likely caused by lack of regularisation at the boundary.

Then, for all 17 subjects the full group-wise optimisation Algorithm 2 was run. The aim of the experiment was to see how well Algorithm 2 converged, and to see the effects of the diffusion imaging. Again we have no gold standard, as we are hypothesising that the parcellation should change to something that's only visible on diffusion images, which have not been manually segmented. We ran the main loop, Algorithm 2 for 5 iterations, by which point the internal loop running Algorithm 1 had converged. Fig. 3 illustrates the movement of the parcellation boundary on a single subject. Once the groupwise registration had converged, the difference in the boundary can be seen.
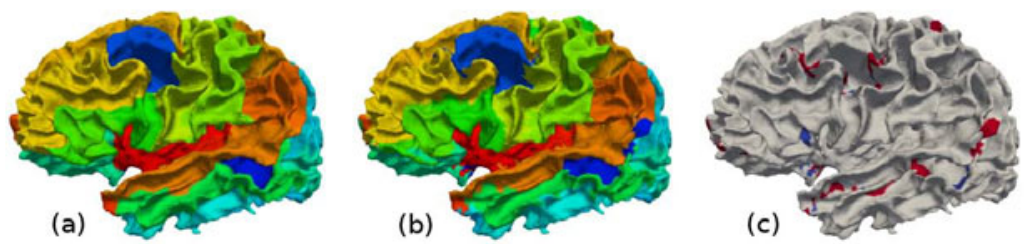

Fig. 3. Results of Algorithm 2 (a) Initial (FreeSurfer) atlas based parcellation. (b) After groupwise optimisation using Algorithm 2 the boundaries have moved. (c) A difference image showing the difference between (a) and (b).

\section{Discussion and Conclusion}

We have demonstrated an algorithm to update a cortical parcellation using diffusion imaging. This work shows promising and interesting results. Importantly it shows that with a simple optimisation scheme, tract based connectivity does indeed alter an atlas based topographical parcellation, implying that a topography 
based parcellation is not entirely concordant at a functional level between individuals. This is not at all surprising given the well described differences between structure, function and cytoarchitecture correlates 62 and may be particularly relevant in functionally driven applications. While we are aware that we have only performed visual inspection, it is already interesting to see that there may be some regions where the boundary only moves a little, indicating a good concordance between structure and connectivity, and regions where the boundary moves a lot. Further work will investigate the reasons for this.

There are many opportunities for improvement of this algorithm, both on a purely technical basis, and on a more philosophical one. We started this work, inspired by the Spherical Demons paper 12. We aimed to compute a force at each vertex along the boundary. The algorithm attaches connectivity lines directly to points to avoid repeatedly searching for the closest line. However, it is difficult to match the resolution of the tractography to the resolution of the spherical mesh. This results in a sparse number (approx 20-30\%) of boundary points having connection data. For these results, we down-sampled the spherical mesh, using larger triangles, and attached the connections to the closest available point. This increased the percentage of points having connections, but effectively reduced the resolution. We need to study the effect of different tract seeding strategies, the number of tracts, and the masks used.

Our optimisation was simple, and could certainly be improved. We have not yet implemented any form of regularisation which leads to small islands appearing in the parcellation. This could be remedied by maintaining an explicit boundary model, enforcing boundaries are not broken. Alternatively, rather than optimise on a point by point basis, a regular grid of control points could be used. Furthermore, the connectivity histogram is complex, and could be smoothed [9] or a more global optimisation scheme adopted.

The question of how closely structure and function should match, on an individual and group-wise basis, is an interesting one. It has been shown that there can be significant differences between subjects [1]. It may be better to simply deal with each subject individually. The voxel based methods described in 62 . provide a way to search for clusters, purely within the tractography information with no a priori knowledge of the existence of any parcellation. A combination of an existing parcellation from an atlas, and also a clustering technique on the tracts rather than a clustering on the connectivity histogram may provide a stronger indicator of distinct regions.

For applications where the scale of interest is large, such as thickness measurements over large regions of the cortex, then deriving fine grained boundary detail may provide little statistical difference. Techniques such as this and 62 may provide greater insight in areas where from a clinical perspective there is a need to sub-divide a given region. Another interesting area of study would be in longitudinal analysis. Over time, changing regional boundaries may confound atlas based parcellation algorithms. Using diffusion imaging and a model of how a disease process might affect tracts over time, may provide a way for connectivity information to iteratively update the parcellation boundaries. 
In conclusion, we have demonstrated a prototype framework to iteratively update an atlas based parcellation using diffusion imaging. Initial results prove promising, and potentially open up many more areas of research.

\section{References}

1. Amunts, K., Malikovic, A., Mohlberg, H., Schormann, T., Zilles, K.: Brodmann's areas 17 and 18 brought into stereotaxic space-where and how variable? Neuroimage 11(1), 66-84 (2000)

2. Beckmann, M., Johansen-Berg, H., Rushworth, M.F.S.: Connectivity-based parcellation of human cingulate cortex and its relation to functional specialization. J. Neurosci. 29(4), 1175-1190 (2009)

3. Cathier, P., Mangin, J.F.: Registration of cortical connectivity matrices. In: Conference on Computer Vision and Pattern Recognition Workshop, CVPRW 2006, p. $66(17-22,2006)$

4. Fischl, B., Dale, A.M.: Measuring the thickness of the human cerebral cortex from magnetic resonance images. PNAS 97(20), 11050-11055 (2000)

5. Fischl, B., van der Kouwe, A., Destrieux, C., Halgren, E., Ségonne, F., Salat, D.H., Busa, E., Seidman, L.J., Goldstein, J., Kennedy, D., Caviness, V., Makris, N., Rosen, B., Dale, A.M.: Automatically parcellating the human cerebral cortex. Cereb Cortex 14(1), 11-22 (2004)

6. Johansen-Berg, H., Behrens, T.E.J., Robson, M.D., Drobnjak, I., Rushworth, M.F.S., Brady, J.M., Smith, S.M., Higham, D.J., Matthews, P.M.: Changes in connectivity profiles define functionally distinct regions in human medial frontal cortex.. Proc. Natl. Acad. Sci. U. S. A. 101(36), 13335-13340 (2004)

7. Park, H.J., Kim, J.J., Lee, S.K., Seok, J.H., Chun, J., Kim, D.I., Lee, J.D.: Corpus callosal connection mapping using cortical gray matter parcellation and DT-MRI. Hum. Brain Mapp. 29(5), 503-516 (2008)

8. Rettmann, M.E., Han, X., Xu, C., Prince, J.L.: Automated sulcal segmentation using watersheds on the cortical surface. Neuroimage 15(2), 329-344 (2002)

9. Roca, P., Rivière, D., Guevara, P., Poupon, C., Mangin, J.F.: Tractography-based parcellation of the cortex using a spatially-informed dimension reduction of the connectivity matrix. Med. Image Comput. Comput. Assist Interv. 12(Pt. 1), 935$942(2009)$

10. Thompson, P.M., Schwartz, C., Toga, A.W.: High-resolution random mesh algorithms for creating a probabilistic 3D surface atlas of the human brain. Neuroimage 3(1), 19-34 (1996)

11. Tournier, J.D., Calamante, F., Connelly, A.: Robust determination of the fibre orientation distribution in diffusion MRI: non-negativity constrained super-resolved spherical deconvolution. Neuroimage 35(4), 1459-1472 (2007)

12. Yeo, B.T.T., Sabuncu, M.R., Vercauteren, T., Ayache, N., Fischl, B., Golland, P.: Spherical demons: fast diffeomorphic landmark-free surface registration. IEEE Trans. Med. Imaging 29(3), 650-668 (2010) 